\title{
Plagiaire et faussaire : des auteurs pervertis
}

\author{
Hélène Maurel-Indart
}

\section{OpenEdition}

Journals

Édition électronique

URL : http://journals.openedition.org/recherchestravaux/1321

DOI : 10.4000/recherchestravaux.1321

ISSN : 1969-6434

\section{Éditeur}

UGA Éditions/Université Grenoble Alpes

\section{Édition imprimée}

Date de publication : 15 mai 2004

Pagination : 205-212

ISBN : 2-9518254-3-9

ISSN : 0151-1874

\section{Référence électronique}

Hélène Maurel-Indart, "Plagiaire et faussaire : des auteurs pervertis », Recherches \& Travaux [En ligne], 64 | 2004, mis en ligne le 20 mai 2019, consulté le 08 septembre 2020. URL : http://

journals.openedition.org/recherchestravaux/1321; DOI : https://doi.org/10.4000/recherchestravaux. 1321 
Hélène MAUREL-INDART

Université François Rabelais-Tours

\section{Plagiaire et faussaire: des auteurs pervertis}

Prenons l'hypothèse qu'il existe dans le domaine de la création littéraire une relation idéale, transparente, entre l'auteur et son œuvre. La transparence relationnelle entre un auteur et sa création s'entendrait dans le sens d'une adéquation exacte entre le scriptor du texte et l'auctor. Jean-François Jeandillou ${ }^{\mathrm{I}}$ précise ainsi les dénominations de Barthes:

$X$ étant le nom du scriptor réel, c'est-à-dire de celui qui, matériellement, tient la plume en main, $Y$ peut alors se définir comme le nom de l'auctor, c'est-à-dire de l'autorité, du garant qui assume la responsabilité apparente d'une œuvre dont il n'est que le signataire, mais non le fabricateur véritable.

Je rêve donc d'une bibliothèque idéale où chaque livre me renverrait en toute certitude, en toute sérénité, à un être bien identifié, tout à la fois signataire et fabricant. Le lecteur, en quête d'auteur, c'est-à-dire de guide, de modèle ou de parent, bref, de référent intellectuel, moral ou affectif, pourrait ainsi s'en remettre à une œuvre formant un tout cohérent, en dépit même de ses contradictions et de ses ruptures. Il trouverait même dans ces contradictions et ces erreurs la confirmation de ce qu'il recherche: l'univers en construction, et finalement achevé par la force des choses, d'une entité humaine en laquelle il puisse se reconnaître, reconnaître en cet autre ce qui est en lui-même indicible ou simplement confus. Il y puiserait comme en un autre soi-même une justification de sa propre existence. Ou bien, il pourrait le rejeter en partie ou en totalité, pour aller vers un autre rayon de la bibliothèque, vers une autre lettre de l'alphabet qui le conduirait vers un autre univers lui permettant de réaliser par identifications et rejets successifs sa propre quête de soi. La littérature nous doit bien cela.

I. Esthétique de la mystification, Tactique et stratégie littéraires, Paris, Minuit, coll. "Propositions", 1994, P. 72-73. 
Mais cette bibliothèque idéale n'aurait sans doute rien de bien humain. Il y manquerait tous les travers et les faiblesses de la nature humaine. Ce serait oublier, en effet, tous ceux que l'auteur de Supercheries littéraires a classés parmi les auteurs fictifs, virtuels, apparents, prétendus, présumés, supposés ${ }^{2}$. La relation d'adéquation entre le scriptor et l'auctor est en réalité souvent pervertie, pour des raisons diverses.

Ainsi, le goût du jeu ou de la provocation incite certains scriptores à se cacher derrière des pseudonymes, voire des hétéronymes ${ }^{3}$ ou même, dans le cas de Romain Gary, d'un vrai-faux hétéronyme, puisque la personne pour laquelle se fait passer le scriptor existe bien en chair et en os mais sous un faux nom!

La professionnalisation de l'activité d'écriture, depuis le XIX' siècle, est une autre cause de la perversion de la relation auteur-œuvre. Elle a poussé certains auteurs à succès à déléguer à des collaborateurs - "nègres " - leur travail de documentation ou même de rédaction. On apprend ainsi, à la faveur d'un plagiat commis par le scriptor, que le signataire du livre n'en est pas luimême l'auteur. Parmi d'autres, $\mathrm{M}^{\mathrm{gr}}$ Gaillot ${ }^{4}$ connut ce triste sort, ou encore une personnalité comme Jean Guitton, compromis par ses coauteurs, les frères Bogdanov, dans un ouvrage d'entretiens truffé de plagiats's

Les plagiaires viennent donc aussi pervertir la relation d'appartenance d'une œuvre à son auteur, pour des motifs psychologiques ou financiers, les uns étant poussés par l'appât du gain, d'autres par le besoin de reconnaissance ou de gloire littéraire, d'autres enfin, de manière plus ou moins consciente et d'autant plus tragique, par impuissance créatrice.

Une belle nouvelle de Didier Daeninckx intitulée "Le Manuscrit trouvé à Sarcelles ", dans son recueil Passages d'enfer (Denoël, 1998), relate la mésaventure d'un membre de l'Académie qui voit tourné en dérision par ses pairs le manuscrit d'un roman qu'il avait oublié dans un taxi et qu'un autre trouva bon de publier en son propre nom, au lieu de le rendre à son propriétaire. Le plagiaire, Freddy Moerdeley, au chômage, avait, plus que la fortune, espéré la gloire et la reconnaissance avec la publication de cet ouvrage:

2. Jean-François Jeandillou, Supercheries littéraires, La vie et l'ceuvre des auteurs supposés, Droz, Genève, 200I, p. 471-474.

3. "Nom donné (ou prêté) par le scriptor à un autre imaginaire. L'imposition du nom va ici de pair avec l'invention d'une biographie, la constitution d'un "portrait de caractère", la production de prétendus documents (manuscrits ou iconographiques) etc." Jean-François Jeandillou, Esthétique de la mystification, op. cit., p. 80.

4. La Dernière Tentation du diable (Édition numéro 1, 1998) est un plagiat du Retour du diable de Paul Ariès (Éd. Golias, 1997).

5. Dieu et la science (Grasset, 1991) a été qualifié de contrefaçon de La Mélodie secrète de Trinh Xuan Thuan par la cour d'appel de Paris le 26 mai 1992. 
La prose de l'inconnu l'impressionnait [...]. Tout lui paraissait aller de soi, et il s'avoua que c'était ainsi qu'il aurait aimé écrire : l'inconnu s'accordait à sa voix.

Tandis que le plagiaire pervertit la relation auteur-texte en signant de son nom ce qu'un autre a écrit, le faussaire, à l'inverse, exerce sa perversion en signant d'un nom d'auteur connu une œuvre qu'il a lui-même fabriquée, en reproduisant le style de cet auteur. Le faussaire agit par jeu, pour tromper les experts, ou dans un but lucratif, puisqu'il pourra vendre au prix fort une œuvre qui, signée de son nom, aurait été peu cotée. En Allemagne, le cas Konrad Kujau, auteur des faux carnets d'Hitler, a marqué les esprits ${ }^{6}$. Luimême, ainsi que l'hebdomadaire Stern qui publia en 1983 les carnets personnels du Führer, se firent une fortune éphémère de cette exclusivité, jusqu'à la dénonciation deux semaines plus tard de la supercherie par les spécialistes de la période. Après ses quatre ans et demi de prison, la passion de la contrefaçon poussa l'homme à peindre des faux Dali et des faux Chagall en les marquant d'une double signature, la sienne et celle de l'artiste. Peut-on voir dans le signalement explicite de la double origine de l'œuvre l'expression d'une confusion identitaire chez le contrefacteur, qui dépasse le simple souci de profit financier?

Les exemples de faux abondent dans le domaine des Beaux-Arts. En littérature, on doit, entre autres, aux faussaires la fameuse affaire de $L a$ Chasse spirituelle faussement attribuée à Rimbaud...

Les cas de suppositions d'auteur ajoutent à la confusion de notre bibliothèque de rêve, puisqu'ils consistent à diffuser une œuvre sous le nom d'une personne qui a priori aura plus de succès que si elle avait été signée de son véritable auteur et de berner ainsi les experts. C'est bien l'explication donnée par Jean-François Jeandillou pour "l'invention du Noyer de Maguelonne", du supposé André Frédoli, qui «avait pour but de prendre en défaut la vigilance et la clairvoyance des experts, car seuls étaient dans le secret les plus proches amis du faussaire ${ }^{7}$ ".

Je souhaiterais à ce propos souligner l'ambiguïté du terme de faussaire qui peut qualifier à la fois l'auteur d'une œuvre faussement attribuée à un autre artiste bien réel et connu - on parle alors de forgerie -, et l'auteur d'une œuvre attribuée à un faux auteur, inventé de toute pièce - c'est la supposition d'auteur. Dans le second cas de supposition d'auteur, seule la signature

6. Voir sur cette affaire l'article de Lucas Delattre dans Le Monde du is septembre 2000, et le film d'Helmut Dielt, Shtonk (1993).

7. Supercheries littéraires, op. cit., p. 43. 
est fausse; le scriptor, en revanche, fabrique bien une œuvre véritable, inspirée certes d'un courant ou d'un style particulier, mais non reproduite exactement à partir des caractéristiques de style d'un auteur réel, comme dans le cas du faux/forgerie.

La supposition d'auteur se distingue donc à la fois du plagiat et de la forgerie, dans la mesure où il $\mathrm{y}$ a création d'un texte nouveau. Reste au chercheur à relier cette œuvre attribuée à un auteur supposé au véritable auteur et à restituer les circonstances de toute cette machinerie qui accompagne ce genre d'affaire. En revanche, ni le plagiaire ni le faussaire ne produisent $-a$ priori - une œuvre originale dans un style personnel. Peut-on même parler d'auteur, tant il est vrai que ces figures paradoxales d'auteur heurtent la doxa, l'opinion commune que l'on se fait d'un auteur et de sa relation avec son œuvre, telle que nous l'avons définie comme principe de départ?

Le faussaire est un écrivain sans style personnel, le plagiaire est un écrivain sans œuvre personnelle. Le faussaire, en effet, reprend exactement les caractéristiques de style d'une œuvre pour en reproduire une variante; quant au plagiaire, il ne fait que recopier, en la démarquant, une œuvre déjà existante. Pour l'un comme pour l'autre, le terme d'écrivain est à prendre dans son sens le plus faible. L'un comme l'autre ne font acte d'écrire qu'au sens physique du terme, que ce soit en imitant scrupuleusement - le faussaire - ou en recopiant servilement - le plagiaire. L'un et l'autre pervertissent la notion même d'écrivain, en même temps que d'œuvre.

A moins que certains faux ne puissent eux-mêmes accéder au statut d'œuvre. Ainsi, pourquoi un faux Modigliani ne vaudrait-il pas un vrai Modigliani? En soi, un faux d'excellente qualité ne se distingue pas d'un authentique tableau d'auteur. La moindre valeur ne tient qu'à l'origine du tableau, non pas à sa qualité intrinsèque. Il en est de même pour un texte, selon Jean-François Jeandillou:

Faut-il comprendre par là qu'un texte perd toute sa «valeur» littéraire sous prétexte que l'auteur a maquillé son identité? On ne pourrait alors juger d'un livre que d'après la personne même qui l'écrit ${ }^{8}$.

Dès lors que La Chasse spirituelle perd sa valeur d'œuvre rimbaldienne, perdrait-elle toute valeur littéraire? Ne pourrait-elle pas être lue pour ellemême, indépendamment de son origine? Y aurait-il donc des œuvres bien nées, susceptibles de recueillir l'approbation de la communauté littéraire, en dehors de leur seul intérêt anecdotique, tandis qu'en marge seraient maintenus à l'écart des textes orphelins, bâtards ou, en un mot, d'origine douteuse?

8. P. 494 . 
Que penser d'une telle discrimination, liée uniquement à l'origine? Si le faux ne peut, à juste titre, être apprécié selon les mêmes critères qualitatifs que l'original, sur un plan purement esthétique, c'est que la source d'inspiration est chez un autre que celui qui le fabrique. Quelle que soit l'extrême habileté du scriptor, elle ne s'exerce que sous l'influence directe et nécessaire d'un autre artiste, à l'origine d'un style original, personnel et nouveau. Cette virtuosité n'apporte aucune plus-value, l'objectif étant justement de faire passer une ouvre pour celle d'un autre. Dans ce cas précis, l'influence n'est pas un point de départ, à partir duquel un nouvel univers stylistique pourra émerger. Le faussaire ne fait que reproduire ce qui sans l'autre n'aurait jamais été. Le faux, coupé de son origine, est une œuvre sans avenir. Il représente une sorte d'impasse dans l'histoire littéraire. Pour preuve, le faussaire ne revendique pas le statut d'auteur; il se cache et ne doit sa fortune qu'à son anonymat. Il n'existe qu'en coulisse pour recueillir les fruits de sa supercherie. Quant à son œuvre, elle n'est ni la sienne, ni celle de l'autre. Elle est, non pas orpheline, mais clone privé de sens.

Comme le faussaire, le plagiaire, autre figure paradoxale d'auteur, n'a pas d'œuvre propre. Mais contrairement à lui, il a un nom dont il signe l'œuvre des autres. Il se fait passer pour auctor, auteur perverti en réalité, qui, tel la cocotte, biaise, emprunte les chemins de traverse, en singeant les manières de la femme du monde. Le plagiaire a été mal doté par la nature qui lui a donné le désir de plaire, par l'écriture, sans lui en donner les moyens. Tel une Traviata de la littérature, il est touché par l'amour d'écrire, sans jamais en trouver la voie. Dévoyé, il recourt à l'artifice du plagiat pour accéder à la reconnaissance littéraire et réaliser son rêve d'auteur. Au risque de perdre sa propre identité dans cet amour impossible pour l'œuvre d'un autre qui devient le sens même de son existence. Telle encore La Traviata, il subit par une figure d'autorité - le critique littéraire, voire le juge - le rappel à l'ordre moral qui lui interdit une telle relation avec l'objet aimé. La perversion de la relation auteur-œuvre est dénoncée au nom d'une conception idéale de la création littéraire.

Au nom d'une relation légitime, c'est-à-dire univoque et transparente, entre l'auteur et son œuvre, le scriptor authentique ou l'un de ses représentants - un lecteur assidu ou l'ayant droit, appuyé par l'éditeur, le juge ou la presse littéraire - met fin aux agissements de l'auctor usurpateur. Le patrimoine littéraire, de même que le patrimoine familial dans La Traviata, doit ainsi être sauvegardé. 
Il importe tout de même de signaler qu'au nom d'une littérature authentique, de fort mauvais procès pour plagiat visent en réalité à défendre une littérature conforme à certains intérêts moraux, sans rapport avec l'authenticité de l'œuvre et l'intérêt des lecteurs. La campagne de presse virulente qui mit fin à la vie littéraire de Yambo Ouologhem, accusé d'avoir plagié, dans son roman, Le Devoir de violence (Le Seuil, 1969, prix Renaudot), Le Dernier des Justes d'André Schwarz-Bart, se déchaînait davantage contre la dénonciation de l'Afrique précoloniale et l'indépendance d'esprit de l'écrivain malien.

A chaque accusation de plagiat, il est prudent de s'interroger sur les enjeux qui peuvent être en cause. Outre les raisons d'ordre moral ou politique, il en est d'autres, de nature plus matérielle: la protection, non pas de l'œuvre, mais plutôt de l'héritage des ayants droit, au nom d'un droit moral interprété de manière abusive. On a vu récemment les descendants de Victor Hugo s'insurger contre l'auteur d'une suite des Misérables, alors qu'il s'agit là d'un genre littéraire depuis longtemps pratiqué et parfaitement légal concernant des œuvres tombées dans le domaine public.

On voit bien que tantôt le plagiaire sert de bouc émissaire à une société soucieuse de ses intérêts moraux et patrimoniaux, tantôt au contraire il est un véritable parasite de la littérature. Seuls l'examen de l'œuvre incriminée et l'évaluation de l'étendue et de la nature de l'emprunt permettent de trancher entre les deux catégories de plagiaires. Afin de confirmer les conclusions de l'analyse comparative, il est quelquefois utile de comprendre l'intention dans laquelle a été écrit le texte suspect. En effet, il existe un profil-type de plagiaire pour lequel la littérature n'est pas une fin en soi, mais seulement un moyen de reconnaissance et de rayonnement personnel. On explique ainsi l'engouement de certains hommes politiques ou de certaines personnalités médiatiques pour l'écriture ou plus exactement pour la publication d'ouvrages littéraires, quelquefois des romans, mais le plus souvent des biographies, des essais ou des mémoires dont le travail de préparation - au sens le plus large - peut être délégué. La fin justifiant les moyens, le plagiat s'impose finalement comme un moyen pratique de réaliser rapidement un livre. On s'étonnera par exemple, chez tel auteur, de la régularité excessive de publication, en contraste avec l'ampleur du travail exigé pour la réalisation d'un ouvrage. A lire l'inépuisable bibliographie qu'Alain Minc insère à la fin de sa biographie imaginaire de Spinoza ${ }^{9}$, on comprend mieux la condamnation pour contrefaçon dont il a fait l'objet. Les vocations subites chez un homme d'affaires sont tout aussi suspectes. Marc Lévy, auteur d'un roman à

9. Spinoza, un roman juif, Gallimard, 1999. 
succès intitulé Et si c'était vrai... ${ }^{\mathrm{IO}}$, est actuellement en procès pour contrefaçon et l'affaire est sérieuse. La surmédiatisation de certains écrivains laisse aussi penser que la littérature n'est plus qu'un moyen de parvenir, sans répondre véritablement à une vocation authentique.

Le contexte intentionnel est donc déterminant, même si l'on sait que certains chefs-d'œuvre ont été exécutés dans l'urgence ou le besoin financier. Pour preuve, bon nombre de romans du XIX' siècle sont parus en feuilletons, au coup par coup, pour faire face à des situations matérielles difficiles et sans être des plagiats pour autant.

La nouvelle de Borges sur Pierre Menard ${ }^{\text {II }}$ pousse à l'extrême cette idée selon laquelle l'intention de l'auteur détermine totalement le statut de l'œuvre: le Quichotte de Cervantès, lorsqu'il est écrit ou plus exactement reconstitué comme de mémoire par un écrivain nîmois du XXe siècle, devient une cuvre nouvelle, porteuse d'un nouveau sens, par le simple fait du décalage chronologique et de l'intention toute originale d'un auteur de faire sienne cette œuvre replacée dans un contexte spatio-temporel différent:

Mon souvenir général du Quichotte, simplifié par l'oubli et l'indifférence, peut très bien être équivalent à la vague image antérieure d'un livre non écrit (p.48).

Pierre Menard est convaincu de donner à lire un livre nouveau. Point de place ici pour le plagiat. Cette histoire symbolique ne vise pas à disculper les plagiaires mais à relativiser la notion de propriété de l'œuvre. L'œuvre appartient à celui qui l'assume, qui la prend en charge à titre personnel, dans une démarche authentique d'assimilation-restitution, avec un sens intrinsèquement lié à sa personnalité, à son histoire, à son univers propre. La preuve en est que les lecteurs du Quichotte de Pierre Menard ne comprendront pas "son » œuvre de la même manière qu'ils ont pu ressentir celle de Cervantès, espagnol du XVII siècle, écrivant dans une langue porteuse du contexte historique et personnel de l'auteur. Pierre Menard, contrairement au plagiaire, ne s'identifie pas à Cervantès en reprenant son texte. Il le réinvente en se démarquant de la personnalité de son prédécesseur et en imposant au texte d'origine sa forme exacte et nécessaire. Point de triche; point de confusion entre l'autre et soi-même, mais une démarche intentionnelle bien identifiée.

Cette démonstration par l'absurde renforce paradoxalement la notion de filiation entre un auteur et son œuvre dont il détermine le sens. Peut être auteur, non pas celui qui simplement écrit l'œuvre, mais celui qui a assez de force et de conviction intérieures pour marquer de son sceau un texte et le soustraire ainsi au hasard:

Io. Robert Laffont, 2000 .

II. "Pierre Menard, auteur du Quichotte», in Fictions, Gallimard, coll. "Folio", 1983. 
Mon complaisant prédécesseur [...] ne repoussa pas la collaboration du hasard: il composait l'œuvre immortelle un peu à la diable, entraîné par la force d'inertie du langage et de l'invention. Moi, j'ai contracté le mystérieux devoir de reconstituer littéralement son œuvre spontanée.

Le plagiaire ne connaît pas cette détermination qui rend l'œuvre produite nécessaire. Il attire le lecteur dans l'impasse d'un texte sans origine propre, bricolé à partir d'objets de récupération auxquels il échoue à donner un nouveau sens, une nouvelle identité verbale. Le dévoyé se fourvoie tragiquement dans l'œuvre des autres, croyant échapper au néant de son identité. 